\title{
A “Potency-Act" Interpretation of Quantum Physics
}

\author{
Alberto Strumia \\ Istituto Nazionale di Alta Matematica Francesco Severi, Rome, Italy \\ Email:info@albertostrumia.it
}

How to cite this paper: Strumia, A. (2021)

A "Potency-Act" Interpretation of Quantum Physics. Journal of Modern Physics, 12 , 959-970.

https://doi.org/10.4236/jmp.2021.127058

Received: April 21, 2021

Accepted: May 21, 2021

Published: May 24, 2021

Copyright (c) 2021 by author(s) and Scientific Research Publishing Inc. This work is licensed under the Creative Commons Attribution International License (CC BY 4.0).

http://creativecommons.org/licenses/by/4.0/

\begin{abstract}
Some considerations are presented on the so called "ontological interpretations" of quantum physics, starting from a remark by Werner Heisenberg on the relation between the probabilistic character of quantum states and the Aristotelian notion of "potency". We show how an interesting revival of the original idea by Heisenberg can be found in the recent scientific and epistemological literature, in order to solve some paradoxical aspects emerging within some of the usual interpretations of quantum physics. Moreover a way seems to be open in order to rediscover the role of Aristotelian-Thomistic notion of "analogy" of "causal agents" operating even in the physical world. The "Potency-Act" interpretation of quantum physics appears aside the role of the Aristotelian notion of "Form" when it is compared with the recent notion of "information" in the context of the physics of "complex systems" and the biology of "living systems".
\end{abstract}

\section{Keywords}

Quantum Mechanics, Quantum Field Theory, Ontological Interpretation, Potency-Act

\section{Introduction}

In the present paper we propose some informal comments on the "ontological" interpretations of quantum physics starting from Heisenberg's suggestion of comparing qualitatively some quantum results with the Aristotelian-Thomistic "Potency-Act" theory.

After presenting a sketch of the most referenced "ontological" interpretations of quantum mechanics, and more generally of quantum physics (here both referenced shortly as QM), in Section 2, we propose to reconsider, as especially "up to date", Heisenberg's suggestion of retaining QM as a sort of contemporary re- 
discovery of Aristotelian "Potency-Act" theory, within the modern context of a mathematical formulation of scientific theories.

In Section 3 we consider and comment an essential quotation from Heisenberg concerning his own idea of establishing a comparison between QM states $\psi$ and their ontological interpretation in terms of Aristotelian "Potency-Act" doctrine.

Section 4 is devoted to some recent contributions by authors following the "Potency-Act" interpretation of QM in the light of the further developments of quantum physics.

In Section 5 we compare the "probabilistic" interpretation of the wave function $\psi$ with the Aristotelian-Thomistic theory of causality, which includes, beside "deterministic" causality, also "probabilistic" causes and even "free" ones, like e.g., human free will. The last matter, which here is not treated, involves also cognitive sciences and anthropology.

Section 6 deals with the example of a simple physical system like a "beam splitter" interferometer in which a single particle (e.g., a photon or an electron) is observed to behave like a coherent wave train, according to QM prediction.

Section 7 applies "Potency-Act" ontological interpretation of QM to vacuum polarization and vacuum fluctuations.

Some concluding remarks are presented in Section 8.

\section{Conventional Interpretations of QM}

In his well known book The Road to Reality. A Complete Guide to the Laws of the Universe [1] at p. 786, Roger Penrose lists six known "ontological" interpretations of $\mathrm{QM}$, i.e.:

1) "Copenhagen",

2) Many worlds,

3) Environmental decoherence,

4) Consistent histories,

5) Pilot wave,

6) New theory with objective $R$.

Moreover, in the last years, a new approach to QM has been opened by quantum information leading to a formalism, even if not to a proper new interpretation, in terms of qubits (see e.g. [2] [3]).

First we will shortly summarize all of them and later we will go back to an original suggestion by Werner Heisenberg.

1) The "Copenhagen" interpretation

The so called "Copenhagen" interpretation, (for a review see, e.g. [4]) initially proposed by Niels Bohr, offers a non-realistic viewpoint according to which QM does not provide a genuine and predictive description of what really is happening within the world, but it allows to make computations about the measurements an observer is able to do thanks to a suitable detecting apparatus. The irreducible wave and particle frames are considered as "complementary". Wave 
function amplitudes $|\psi|$ are related to the local probability density of some particle presence when an observation is done and are responsible of light and matter interference phenomena. Particles are observed as quanta of momentum $p=\hbar k$ non-exactly localizable as points in space and instant events in time, because of Heisenberg uncertainty principle, stating the relations between momentum/ position, and energy/time:

$$
\Delta p \Delta x \geq \frac{1}{2} \hbar, \quad \Delta E \Delta t \geq \frac{1}{2} \hbar .
$$

Then the QM theory appears simply as a theoretical instrument for previsional computation of probabilities of observation and not as a true description of the real world.

2) The "many worlds" or "multiverse" interpretation

The "many worlds" or "multiverse" interpretation (for a review see e.g. [5]) is intriguing but it appears somehow exotic and non-genuinely scientific because of its non-falsifiability (according to K.R. Popper's scientificity criterion, see e.g. [6], part I, chap 2, section 6). Since it assumes that all the possible states $\left|\psi_{n}\right\rangle$ involved into the state function of a system:

$$
|\psi\rangle=\sum_{n} c_{n}\left|\psi_{n}\right\rangle,
$$

are all really existent in some world, So an infinite number of "parallel worlds" are intended to be "actually" existent, each within its "real" universe. All the parallel universes are non-connected components of a whole, so that only one of them may be observed by us. The wave function involves a superposition of many "real" worlds together.

While the "Copenhagen" interpretation is "instrumentalisitc" (in the sense of Popper [6] and T.S. Kuhn [7]) (or even idealistic or "conventionalistic" in the sense of P. Feyerabend, see, e.g. [8]), the "many worlds" interpretation is excessively "realistic". Moreover, the latter interpretation, even if it is fascinating, manifestly violates the "Occam's razor" simplicity principle [9]. which is normally assumed as scientifically basic. Why to suppose the true "actual" existence of infinite worlds when only one of them is observable by us?

3) The "Environmental decoherence" interpretation

The "Environmental decoherence" interpretation includes the interaction with the environment, i.e., what is considered as "external" to the system on which an observer (human or instrumental) is performing measurements.

So the wave function governing the "whole" is required to include, beside the wave function $\left|\psi^{S}\right\rangle$ related to the system on which one is performing measurements and the wave function $\left|\psi^{A}\right\rangle$ describing the instrumental apparatus needed to exploit measurements, also an almost inaccessible wave function for the "external" environment $\left|\psi^{e n v}\right\rangle$, which must be considered no longer as "external".

$$
|\psi\rangle=\left|\psi^{S}\right\rangle \otimes\left|\psi^{A}\right\rangle \otimes\left|\psi^{e n v}\right\rangle
$$


Interactions of the system with the environment and the apparatus appear both as the "causal agent" determining the collapse from coherence of the wave function. Such interaction, which may be even "non-local", is recognized as the physical basis of entanglement [10].

The causal contribution of environment to decoherence needs to be added to the interaction between the measurement apparatus and the system itself. Such approach is "holistic" in the sense that a quantum system cannot be treated as an isolated one, being always influenced by the environment.

4) Consistent histories

The "Consistent histories" interpretation [11] considers QM as an intrinsically probabilistic theory, in which probability does not depend on observational and measurement operations, but is involved in the wave equation itself as a "true law of nature".

Ontologically it appears that "probabilistic causation" is a kind of causality relation in the physical microscopic world, aside "deterministic causation" in the physical macroscopic world. Introduction of "probability" within the causality is no longer interpreted as a lack of causation, or as a consequence of our ignorance about the physical world, but as a new way of actuating causation in QM, aside the "deterministic" causality in classical mechanics.

5) Pilot wave

The "pilot wave" interpretation proposed by Louis De Broglie and later modified by David Bohm attempts a classical-like approach to quantum mechanics suggesting that the particle trajectories are driven by a pilot wave, being oscillations of some matter field $\psi$ just as a photon is a quantum of oscillating electromagnetic fields $E, B$. In Bohm's variant of the theory, a sort of "quantum potential" is guessed and evaluated, the effect of which is to drive particles along definite trajectories leading to interference and wave effects. But the last approach, in its original form, is not compatible with Einstein's relativity.

"The most thoroughly worked-out theory of this type is the pilot wave theory developed by de Broglie and presented by him at the Fifth Solvay Conference held in Brussels in 1927, revived by David Bohm in 1952, and currently an active area of research by a small group of physicists and philosophers.

According to this theory, there are particles with definite trajectories, that are guided by the quantum wave function." [12]

\section{6) New theory with objective $R$}

The "objective reduction" interpretation is looking for an evolutionary continuous process performed by an evolution operator $U$, avoiding jumps about the quantum states of a system.

“Often referred as 'objective- $R$ ' theory. ' $R$ ' stands for the quantum state reduction when a measurement is taking place. Supporters of this interpretation believe that today's quantum mechanics is not here to stay and will be 
replaced by a theory at which $R$ is a real process. Physicists and mathematicians who work in the field, try to balance the inconsistence of $R$ process (discontinuous process, 'jump' from a state to another) with the unitary and simple (at least in Schrödinger's equation) time evolution of the state vector (often denoted as $U$-process)." [13]

\section{Heisenberg's Interpretation of $\mathrm{QM}$ and the Aristotelian "Potency-Act" Theory}

Surprisingly, but not too much, Werner Heisenberg, in his relevant book Physics and Philosophy, suggested the idea that a meaningful "ontological" interpretation of quantum theory could be related, in some sense, to the Aristotelian concepts of "potency" (Latin, "potentia") and therefore "act" (Latin, "actus"), even if the latter is not explicitly referred-to by him. Here are his own words.

"A first and very interesting step toward a real understanding of quantum theory was taken by Bohr, Kramers and Slater in 1924. These authors tried to solve the apparent contradiction between the wave picture and the particle picture by the concept of the probability wave. [...]

The probability wave of Bohr, Kramers, Slater, however, meant more than that; it meant a tendency for something. It was a quantitative version of the old concept of 'potentia' in Aristotelian philosophy. It introduced something standing in the middle between the idea of an event and the actual event, a strange kind of physical reality just in the middle between possibility and reality. Later when the mathematical framework of quantum theory was fixed, Born took up this idea of the probability wave and gave a clear definition of the mathematical quantity in the formalism, which was to be interpreted as the probability wave. It was not a three-dimensional wave like elastic or radio waves, but a wave in the many-dimensional configuration space, and therefore a rather abstract mathematical quantity." [14] (pgs 11-12 [emphasis mine]).

An historical sketch of Heisenberg's "Aristotelian" interpretation of quantum states can be found in [15], where the author explains how (Section 1):

"the relationship between this use and Aristotle's notion was not made by Heisenberg in full detail, beyond noting their common character: that of signifying the system's objective capacity to be found later to possess a property in actuality. For such actualization, Heisenberg required measurement to have taken place, an interaction with external systems that disrupts the otherwise independent, natural evolution of the quantum system. The notion of state actualization was later taken up by others, including Shimony, in the search for a law-like measurement process. Yet, the relation of quantum potentiality to Aristotle's original notion has been viewed as mainly terminological, even by those who used it thus."

The Aristotelian-Thomistic approach suggests that physical entities may be- 
have according two possible ways of existence:

1) Actual existence

2) Potential existence

The actual existence of a particle, or a quantum, which is non-necessarily localizable as a point, but may possess even an extended structure (string, brane, cloud, etc.), is the only one way of existence we may observe and measure.

The potential existence is the way of existence which is "hidden" (i.e., nonobservable) into a wave carrying "information" (the Schrödinger's wave function $\psi)$ about the probability of presence of a particle, which can be "actualized" by a suitable "causal agent". The latter agent may act either by a direct interaction (measurement, environment, interaction with other particles or fields) with the system governed by $\psi$, according to the "conservation laws", or acting disregarding the conservation laws in a non-observable way thanks to the uncertainty Heisenberg's relation.

Among all the "potential states" of matter, the "higher is the probability" for a particle or a system to come to actual existence, the "nearest to act" such potency will be considered (Latin, "potentia proxima"). It must be emphasized that a "potential existence" is different from being nothing and being actually, as a sort of intermediate state. And different levels of potency are allowed to matter. So the quantum vacuum may be considered as an entity which exhibits a "proximate potency" (measured by a probability) to jump to act thanks to Heisenberg's uncertainty, when a suitable "causal agent" is acting on it. "Information" (candidate to be interpreted as the Aristotelian "formal cause") hidden into the wave function requires an agent ("efficient cause") to become operating on potency organizing it as something actually existent.

So the wave function $\psi$ can be interpreted as a mathematical representation of the "nearest potency" states of the quantum system governed by it. Each eigenstate of the Hamiltonian, or the angular momentum, or the spin or other operator describing the system represents an "actual state" of the system as may be measured by an observer thanks to his apparatus, or into which the system collapses interacting with the environment or with any other "causal agent". Collapsing of the wave function represents the passage from "potency" to "act".

i) The "Potency-Act" interpretation of QM is ontologically "realistic" at least as it is realistic the "consistent histories" interpretation. Since it is assumed that probability is intrinsic to the ontology of the "causal agents" acting on a quantum physical system, independently of the measurement and observation instruments. Probability is assumed to be intrinsic to the nature of a microscopic causation process and does not arise as a statistical effect of measurement procedures.

ii) The "many worlds" or "parallel universes" of the Everett's interpretation of QM are admissible in the frame of "Potency-Act" interpretation as worlds existing, but "in potency" and not actually, while only one of them is assumed to be "actually" existent and therefore "observable", as what we usually call "the real 
world".

iii) As a remarkable epistemological consequence it follows that the "Occam's razor" principle which requires to minimize the number of entities and assumptions within a scientific theory, is fully satisfied. No superfluous actual worlds are required: "Entities must not be multiplied without necessity" (Latin, "entia non sunt multiplicanda sine necessitate").

\section{The "Potency-Act" Interpretation after Heisenberg}

Significantly it must be emphasized that recently, about a century after Heisenberg, a "Potency-Act" Aristotelian-Thomistic interpretation of QM has come to the attention of several researchers and philosophers of QM ontology. Among them we may mention, e.g. Ignacio Silva [16], Robert R. Bishop and Joseph E. Brenner [17], Alfred Driessen [18], Boris Bozňjak [19], and others.

According to Silva:

"With this interpretation Heisenberg abandons the Parmenidean/Cartesian materialistic ontology to embrace a new ontology for quantum phenomena: the ontology of act and potency. He moves from the rigid mechanism of Cartesian ontology to a richer Aristotelian ontology. According to Heisenberg, developments in quantum mechanics have given rise to a more subtle concept of reality than that based in classical physics. The univocal notion of being assumed in classical physics should be left aside for an analogical notion of being. This thought opens a path for a new development of the concepts of philosophy of nature of act and potency." [16] (pg 641).

A "classical" suggestion of what happens in a binary quantum system (like the electron spin), thanks to which one can guess what it happens, is provided by a coin tossed on air and later fallen down to ground. When the coin is flying on air its state is undetermined, being "potentially" either "head" or "tail", in a sort of mixture of both states.

Only after its fall onto the ground it reaches an "actual", definite "eigenstate", which may be, with the same probability $\frac{1}{2}$, either "head" or "tail".

The electron spin, as any other binary quantum system, behaves just as a "superposition" (linear combination) of two eigenstates $|\uparrow\rangle$ (up) and $|\downarrow\rangle$ (down):

$$
|\psi\rangle=\alpha_{\uparrow}|\uparrow\rangle+\alpha_{\downarrow}|\downarrow\rangle .
$$

We can interpret such a superposition as a state "in potency" of the electron, which collapses into an "actual" eigenstate ("up" or "down") after an actuating causal process (interaction with other systems, measurement apparatus, environment). So a binary system like the "Schrödinger's cat" is not actually dead and alive at the same time, but is potentially dead or alive before being observed, since it is "actually alive" and "potentially dead" until the poison ampule is broken and vice-versa after the latter event. According to "Potency-Act" interpreta- 
tion of QM no paradoxical situation happens.

Only the universe within which the cat is in its "actual eigenstate" is the "actual universe", while the so called "parallel universe" is "in potency".

Therefore in a "Potency-Act" interpretation of QM we may also assume that "waves", as mathematically described by the wave function $\psi$, involve the states of the system "in potency", while the "particles" (i.e., the extended structures like quanta, strings, branes, clouds, etc.) involve the states of the system "in act". The mathematical formalism of operators, implying the uncertainty principle for non-commuting physical quantities:

$$
\Delta A \Delta B \geq \frac{1}{2}|\langle[A, B]\rangle|,
$$

$[A, B]=A B-B A$ being the commutator, when referred to the conjugate operators of position $x$ and momentum $p$ of a "particle":

$$
\Delta x \Delta p \geq \frac{1}{2} \hbar,
$$

being $[x, p]=i \hbar$, simply declares that the point like representation of a structured system is unphysical, being too simplistic.

\section{Different Types of Causal Agents}

The "realistic" character of probability which is presupposed in the "Potency-Act" interpretation of QM, has its remote ontological foundation in the Aristotelian-Thomistic theory of causality.

In fact, according to the latter physical and metaphysical view of reality, three kinds of behavior of "causal agents" may be observed in nature.

1) The "deterministic" causal agents (Latin, ad unum);

2) The "probabilistic" causal agents (Latin, ut in pluribus or highly probable; ut in paucioribus or less probable);

3) The "free" causal agents (Latin, ad utrumlibet or ambivalent).

The first ones behave like causes in "classical physics" (like forces and fields), according to which the evolution phase trajectory of a system is "bi-univocally" determined assigning the initial (or the boundary) conditions beside the physical laws governing the examined phenomenon.

The second ones behave like causes in "quantum physics" (like quantum potentials and quantum fields), since the effect of their action is non-deterministic and non-univocal, so that the phase trajectories of a system are undetermined and only probability clouds can be estimated. Heisenberg himself says that:

"in modern physics the concept of possibility, that played such a decisive

role in Aristotle's philosophy, has moved again into a central place.” [20]

The third ones behave like "human freedom", being unpredictable in each individual choice which may be indifferently oriented, if it is left alone.

Manifestly according to the "Aristotelian-Thomistic" philosophy of nature, on which the "Potency-Act" interpretation of QM is based, the probability is intrin- 
sic to the system and is not induced by external or subjective factors. Moreover:

"the transition from the 'possible' to the 'actual' takes place as soon as the interaction between the object and the measuring device, and thereby with the rest of the world, has come into play; it is not connected with the act of registration of the result in the mind of the observer." [14] (pgs 54-55).

According to Heisenberg the "causal agent" of the transition from a "potential" to an "actual" state of a system has an objective character and is neither a sort of subjective mental projection of mind, in the sense of idealistic philosophy nor a spontaneous event happening in absence of an "adequate causation".

\section{The Example of the "Beam Splitter" Interferometer}

Let us now apply the "Potency-Act" interpretation of QM to the interference phenomenon happening in a wave "splitter apparatus" like the one shown in Figure 1.

A photon or an electron described by the wave function $\psi$ is incoming into the apparatus (see "IN" in Figure 1, where it splits into two half particles (waves of half amplitude). The transmitted half wave proceeds horizontally with unaltered phase. So the reflected wave which is running down maintains its original phase being submitted to an "internal" reflection. In S1, S2 each one of the coherent half wave meets, at the same distance, an ordinary glass, where is submitted to "external" reflection, which reverses its phase (phase shift of $\pi$ ). Eventually the half waves reach "OUT" where they split a second time into half a half wave, the red wave reflecting "internally", the blue wave reflecting "externally". On "OUT" two observers (detectors) are placed on the horizontal and on the vertical directions.

The horizontal observer detects two superposed waves in phase concordance, while the vertical observer detects nothing since the two superposed waves emerging from the apparatus are in phase opposition and destroy themselves.

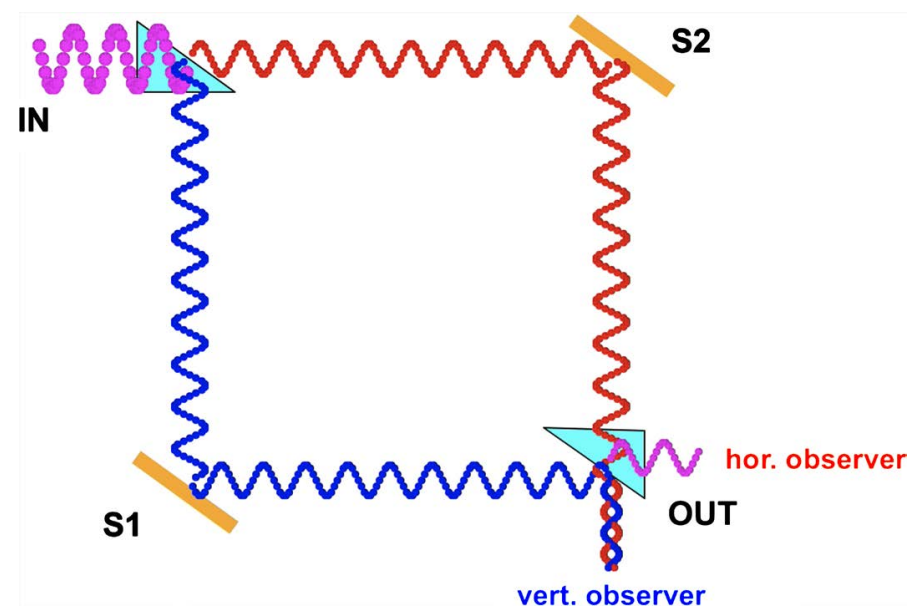

Figure 1. Interference in a photon/electron wave splitter apparatus (click for animation, http://www.albertostrumia.it/sites/default/files/Animations/QMechanics/SplitterOnde.m $\underline{4 \mathrm{v})}$. 
According to the "many worlds" interpretation the photon/electron wave observed by the horizontal detector lives in our world, while the other wave which is not observable by the vertical detector lives in a "parallel universe". A somehow strange situation!

While following a "Potency-Act" interpretation we can say the horizontal observer sees the particle which is "actually" existent, while the vertical observer cannot see what has come back from "act" to "potency", because of the action of a suitable "causal agent" (the different numbers of external and internal reflections along the two ways implying destructive interference). The actual world required is the only one which is observable, while the so called "parallel world" (non-observable) needs no longer to be supposed as actually existing, but only "potentially" existing as hidden into the capabilities of matter.

\section{The Quantum Fluctuation of Vacuum}

Another relevant question involves the "quantum fluctuations of vacuum". It is known that particle-antiparticle pairs (e.g., electron-positron) may emerge from "quantum vacuum" under the "external causal action" of an electromagnetic field.

Therefore people is investigating if the universe may have arisen because of an "internal causal action" arising spontaneously as a quantum fluctuation. Is such an internal action coming from some "informational causal agent" acting from "outside" the material universe, being in itself "immaterial" as any information?

Moreover, does such information play a "teleonomical" role driving the evolution of universe from a vacuum fluctiation towards "attractors" like elementary particles, nuclei, atoms, molecules, stars, galaxies, planetary systems, living beings?

As Boris Kožnjak observed:

"Final causes in fact provide a better ontology for quantum mechanics than spontaneous causation. The idea of 'spontaneity' is unanalyzable and therefore of little use in quantum mechanics.

In addition, it is ontologically sterile in the context of quantum measurement, as shown by a historical and conceptual review of the role of efficient causation in experimental physics." [19]

\section{Conclusions}

We have proposed an introductory sketch on the recent revival of interest in Aristotelian-Thomistic "Potency-Act" theory of matter states (and more generally "entities") in order to provide an intriguing ontological interpretation of quantum physics, as originally was suggested by Werner Heisenberg at the very beginning of quantum mechanics. New perspectives appear especially promising even in order to rediscover the Thomistic doctrine of "analogy of being" (Latin, “analogia entis") [21].

The latter way of investigation, about quantum physics, appears to be com- 
plementary to a parallel rediscovery of the "Form-Matter" Aristotelian-Thomistic doctrine, in the context of information theory and its relations to complexity, self organization and the biology of living systems [22]. Even if it would be, at present, at least arbitrary to identify the Aristotelian "form" with our "information" and the Aristotelian "potency" with "quantum probability" as mathematically described by the wave function $\psi$, the early suggestion by Heisenberg seems today more and more relevant.

\section{Acknowledgement}

I want to thank especially J.E. Brenner for relevant interesting suggestions he proposed to improve the quality of my paper.

\section{Conflicts of Interest}

The author declares no conflicts of interest regarding the publication of this paper.

\section{References}

[1] Penrose, R. (2004) The Road to Reality. A Complete Guid to the Laws of the Universe. Jonathan Cape, London.

[2] Deutsch, D. (2004) It from Qubit. In: Barrow, J., et al., Eds., Science \& Ultimate Reality, Cambridge University Press, Cambridge, 90-102. https://doi.org/10.1017/CBO9780511814990.008

[3] Dür, W. and Heusler, S. (2014) The Physics Teacher, 52, 489. https://arxiv.org/abs/1312.1463 https://doi.org/10.1119/1.4897588

[4] Faye, J. (2019) Copenhagen Interpretation of Quantum Mechanics. https://plato.stanford.edu/archives/win2019/entries/qm-copenhagen

[5] Vaidman, L. (2018) Many-Worlds Interpretation of Quantum Mechanics. https://plato.stanford.edu/archives/fall2018/entries/qm-manyworlds

[6] Popper, K.R. (1992) The Logic of Scientific Discovery. Routledge, London and New York.

[7] Kuhn, T.S. (1996) The Structure of Scientific Revolutions. The University of Chicago Press, Chicago and London.

[8] Feyerabend, P. (1993) Against Method. Verso, London.

[9] Baker, A. (2016) Simplicity. Stanford Encyclopedia of Philosophy. https://plato.stanford.edu/archives/win2016/entries/simplicity

[10] Zeh, H.D. (1995) Decoherence: Basic Concepts and Their Interpretation. https://arXiv:quant-ph/9506020

[11] Griffiths, R.B. (2019) The Consistent Histories Approach to Quantum Mechanics. Stanford Encyclopedia of Philosophy. https://plato.stanford.edu/entries/qm-consistent-histories

[12] Myrvold, W. (2018) Philosophical Issues in Quantum Theory. Stanford Encyclopedia of Philosophy. https://plato.stanford.edu/archives/fall2018/entries/qt-issues

[13] Lazarou, D.K. (2018) Interpretation of Quantum Theory. An Overview. Stanford 
Encyclopedia of Philosophy. https://arxiv.org/abs/0712.3466

[14] Heisenberg, W. (1962) Physics and Philosophy. Harper (Reprint Penguin Classics, 2000), New York.

[15] Jaeger, G. (2017) Philosophical Transactions of the Royal Society A, 375, Article ID: 20160390. https://doi.org/10.1098/rsta.2016.0390

[16] Silva, I. (2013) Werner Heisenberg and Thomas Aquinas on Natural Indeterminism. The Dominican Council, John Wiley \& Sons Ltd., Oxford and Malden.

https://doi.org/10.1111/nbfr.12036

[17] Bishop, R.R. and Brenner, J.E. (2017) Potentiality, Actuality and Non-Separability in Quantum and Classical Physics: Res Potentiae in the Macroscopic World. Stanford Encyclopedia of Philosophy. https://arxiv.org/abs/1801.01471

[18] Driessen, A. (2020) Acta Philosophica, 29, 395-413.

http://philsci-archive.pitt.edu/16265/1/Aristotle and Quantum Mechanics.pdf

[19] Kožnjak, B. (2020) Journal for General Philosophy of Science, 51, 459-480. https://doi.org/10.1007/s10838-020-09500-y

[20] Heisenberg, W. (1960) Wort und Wirklichkeit, 1, 32-62.

[21] Strumia, A. (2007) Acta Biomedica, 78, 32-38. https://www.mattioli1885journals.com/index.php/actabiomedica/article/view/4608

[22] Strumia, A. (2020) From Fractals and Cellular Automata to Biology. Information as Order Hidden within Chance. World Scientific, Singapore.

https://www.worldscientific.com/worldscibooks/10.1142/11743

https://doi.org/10.1142/11743 